\title{
Compreendendo a Influência da Liderança nas Iniciativas de Melhoria de Processo de Desenvolvimento de Software
}

\author{
Alessandra Zoucas ${ }^{1,2}$, Marcello Thiry ${ }^{1}$, Cristiano Cunha ${ }^{2}$ \\ ${ }^{1}$ Universidade Federal de Santa Catarina (UFSC) \\ Programa de Pós-Graduação em Engenharia e Gestão do Conhecimento (EGC) \\ Laboratório de Liderança \& Gestão Responsável (LGR) \\ Florianópolis - SC - Brasil \\ ${ }^{2}$ Universidade do Vale do Itajaí (UNIVALI) - Campus São José \\ Ciência da Computação - Rodovia SC 407, km 04, São José - SC - Brasil. \\ \{alessandrazoucas, marcello.thiry, 01cunha\}@gmail.com
}

\begin{abstract}
Studies on factors influencing Software Process Improvement initiatives (SPI) has revealed that in addition to technological factors, human and social factors also interfere in their success. An investigation to understand the influence of leadership initiatives in SPI is important to identify alternatives to SPI programs so they can be managed more effectively. This paper presents a systematic mapping on leadership, specifically in SPI initiatives. The paper presents the selected publications with emphasis on findings related to leadership initiatives in SPI. With this work, we found that there is still space in the literature for research specifically on the influence of leadership in SPI initiatives, providing an opportunity for contributions that will be explored in future work by the authors.
\end{abstract}

Resumo. Estudos sobre fatores que influenciam as iniciativas de melhoria de processo de software (MPS) tem revelado que além dos fatores tecnológicos, fatores humanos e sociais também interferem em seu sucesso. Uma investigação para compreender a influência da liderança nas iniciativas MPS é relevante para identificar alternativas para os programas de MPS poderem ser geridos de forma cada vez mais efetiva. Este artigo apresenta um mapeamento de estudos relativos à liderança, especificamente em iniciativas de MPS. O trabalho apresenta as publicações estudadas, dando ênfase nos achados referentes à liderança nestas inciativas. Com este trabalho, foi possivel verificar que ainda existe espaço na literatura para uma pesquisa especificamente sobre a influência da liderança em iniciativas de MPS, oferecendo uma oportunidade para contribuições que serão exploradas pelos autores em trabalhos futuros.

\section{INTRODUÇÃO}

A Melhoria de Processo de Software (MPS) baseada em Modelos de Capacidade e Maturidade de Processo "tem se tornado uma força importante na indústria de software global" [Card, 2004]. Esta afirmação continua válida e pode ser confirmada pela procura 
crescente das organizações em reconhecer seus esforços de melhoria por meio de avaliações formais com base em modelos reconhecidos tanto nacionalmente quanto internacionalmente [Travassos e Kalinowski, 2010; SEI, 2010; SEI, 2012; SOFTEX, 2012]. Contudo, a quantidade de organizações que adotam esses modelos ainda é uma parcela reduzida da população total de organizações de software [Staples et al., 2007 apud Montoni e Rocha, 2011]. Isto pode ser atribuído ao fato de que melhorar processos de modo efetivo não é uma atividade trivial, sendo complexa e intensa em conhecimento [Zahran, 1998 apud Montoni, 2008].

Organizações envolvidas com desenvolvimento de software são classificadas como Organizações Intensivas em Conhecimento [Swart e Ninnie, 2006; Alvesson, 2004], pois apresentam características diferenciadas das organizações tradicionais pelo fato de criar e disseminar conhecimentos continuamente. Seus profissionais devem saber trabalhar com dados e transformá-los em informação necessária, bem como analisar tais informações e interpretá-las para obter o conhecimento [Fialho et al., 2010].

A melhoria de processo de desenvolvimento de software também tem como uma de suas características, a execução de atividades de natureza cognitiva, com foco no trabalho intelectual dos envolvidos criando e disseminando conhecimentos continuamente. Uma característica da melhoria de processo de desenvolvimento de software é ser conduzida por especialistas em MPS e pelos colaboradores das organizações que buscam a melhoria de processo de desenvolvimento de seus produtos de software. Desta forma, os envolvidos são pessoas com alta capacidade intelectual e que, tipicamente, apresentam formação acadêmica elevada. Contudo, a formação acadêmica não é suficiente para o sucesso da melhoria de processo de software, ela também depende da capacidade das organizações superarem barreiras críticas que têm impedido a larga adoção de modelos para melhoria de processo [Montoni, 2008]. A compreensão sobre a influência de tais barreiras críticas em iniciativas de MPS é fundamental para apoiar a gerência dos programas de melhoria de processo de desenvolvimento de software.

Com o propósito de identificar fatores de sucesso em iniciativas de melhoria de processo de desenvolvimento de software em contextos específicos uma pesquisa exploratória foi conduzida e os trabalhos encontrados têm demonstrado que as iniciativas de MPS são de caráter sócio-cultural, tecnológico e organizacional [Rainer e Hall, 2002; Rainer e Hall, 2003; Niazi, Wilson e Zowghi, 2006]. Outros trabalhos ainda apontam que, embora a área de Engenharia de Software seja de natureza técnica, existem falhas de natureza humana e social em programas de MPS [Santos et al., 2011; Montoni e Rocha, 2011]. Nestes estudos, é fortemente evidenciado que questões nãotecnológicas com influência direta sobre iniciativas de MPS são o apoio efetivo da alta gerência, capacitação e a conscientização dos membros da organização quanto aos benefícios obtidos com a MPS.

Essas questões são diretamente relacionadas com a capacidade de influenciar pessoas para atingir um propósito comum, conforme o conceito de liderança descrito por Northouse (2004) e aos papéis do líder organizacional estabelecidos por Quinn (2003) conforme apresentado na tabela 1. 
Tabela 1. Papéis do líder em seis categorias. Adaptado de Quinn (2003)

\begin{tabular}{|l|l|}
\hline $\begin{array}{l}\text { Categorias do } \\
\text { Papel do líder }\end{array}$ & Características do papel do líder \\
\hline $\begin{array}{l}\text { Realizador de } \\
\text { Metas }\end{array}$ & $\begin{array}{l}\text { Mantém as metas estabelecidas para a unidade } \\
\text { Obtém foco da unidade para atender aos objetivos esperados } \\
\text { Mantém claro o papel da unidade } \\
\text { Esclarece as prioridades e a direção da unidade } \\
\text { Antecipa problemas de fluxo de trabalho, evita crise } \\
\text { Traz um sentido de ordem e coordenação na unidade. }\end{array}$ \\
\hline Mentor & $\begin{array}{l}\text { Mostra empatia e preocupação no trato com os subordinados } \\
\text { Trata cada indivíduo de maneira sensível e gentil } \\
\text { Mostra preocupação com as necessidades dos subordinados }\end{array}$ \\
\hline Facilitador & $\begin{array}{l}\text { Facilita a obtenção de consenso na unidade de trabalho } \\
\text { Havendo diferenças fundamentais entre os membros do grupo, então trabalha } \\
\text { participativamente para resolvê-los } \\
\text { Desenvolve resolução consensual para diferenças expressadas abertamente }\end{array}$ \\
\hline Monitorador & $\begin{array}{l}\text { Mantém alinhado o controle logístico } \\
\text { Monitora o cumprimento das regras } \\
\text { Compara os registros, relatórios e assim detecta discrepâncias }\end{array}$ \\
\hline Intermediador & $\begin{array}{l}\text { Exerce influência nos níveis mais altos da organização } \\
\text { Influencia nas decisões tomadas em níveis mais altos da organização } \\
\text { Tem acesso à pessoas dos níveis mais altos da organização } \\
\text { Persuasivo e vende novas ideias para níveis mais altos da organização }\end{array}$ \\
\hline Inovador & $\begin{array}{l}\text { Traz ideias criativas. } \\
\text { Propõe experiências com novos conceitos e procedimentos } \\
\text { Resolve problemas com solução criativa e inteligente } \\
\text { Procura por inovações e melhorias em potencial }\end{array}$ \\
\hline
\end{tabular}

Para as Organizações Intensivas em Conhecimento, a presença de um líder capaz de facilitar a expansão das capacidades de compreender complexidades e de esclarecer visões [Senge, 2008] é altamente significativa. As Organizações Intensivas em Conhecimento necessitam de um estilo de liderança diferenciado. Os líderes devem contribuir com mudanças organizacionais necessárias à sua adaptação aos novos cenários [Kotter 1997; Senge, 1990]. Entretanto, trabalhos científicos vêm afirmando que a literatura sobre liderança não foi suficientemente analisada no estudo da criação de conhecimento organizacional [Gourlay, 2006; Lado e Wilson, 1994; Tsoukas, 1996 apud Krogh; Nonaka e Rechsteiner, 2011]. Estes autores discutem que a pesquisa empírica sobre liderança tende a apresentar uma visão limitada sobre o processo do conhecimento. Portanto, uma pesquisa voltada para compreensão da influência da liderança nas iniciativas de melhoria de processo de desenvolvimento de software tem se mostrado relevante e ainda não foram relatados resultados de investigações que estudem especificamente a influência da liderança nas organizações durante a execução de um programa de MPS.

Neste sentido, o presente artigo apresenta os resultados de um mapeamento sistemático da literatura [Kitchenham, 2004] com o objetivo de investigar como pesquisas sobre iniciativas de melhoria de processo de software abordam a questão da Liderança, sem restrições ao tipo de liderança. Para isto, foi realizado um mapeamento sistemático em bibliotecas digitais conveniadas com o Portal da CAPES ${ }^{1}$. As bibliotecas

\footnotetext{
${ }^{1}$ www.periodicos.capes.gov.br/
} 
escolhidas foram SCOPUS $^{2}$ e Web of Science ${ }^{3}$, reconhecidas internacionalmente por publicações internacionais de qualidade. Também foi realizada uma busca nos anais do Simpósio Brasileiro de Qualidade de Software (SBQS) publicado em 2011. Esta busca foi manual por não existir uma biblioteca digital que possibilite esta pesquisa automática e, portanto, foi realizada apenas nas publicações mais recentes.

O artigo está dividido em 4 seções, incluindo esta introdução. A segunda Seção apresenta os procedimentos metodológicos adotados na condução do trabalho. Os resultados analisados estão registrados na Seção 3 e as considerações finais do trabalho são discutidas na Seção 4.

\section{Procedimentos Metodológicos}

A condução do mapeamento sistemático sobre a relevância da liderança em iniciativas de melhoria de processo de software foi realizada com base no trabalho de Kitchenham (2004), tendo sido definida em 3 etapas: Planejamento, Execução e Avaliação.

A fase de planejamento teve como objetivo montar um protocolo de busca que relaciona as informações utilizadas para a pesquisa, permitindo sua reutilização futura e oferecendo oportunidade para acompanhar a evolução da literatura sobre o tema estudado de forma padronizada. As perguntas de pesquisa consideradas neste protocolo foram:

\section{- Questão principal:}

- Qual a influência da liderança nas iniciativas de melhoria de processo de desenvolvimento de software?

\section{- Questões secundárias:}

1) Quais categorias dos papeis do líder organizacional descritos por Quinn (2003) tem relação com fatores de sucesso em iniciativas de melhoria de processo de software?

2) Qual é a visão dos pesquisadores que investigam sobre fatores de sucesso em iniciativas de melhoria de processo de software quando avaliam a liderança em tais iniciativas?

A Tabela 2 apresenta os principais componentes do protocolo utilizado para o mapeamento sistemático. A string de busca foi refinada a partir de strings iniciais mais abertas que retornavam uma quantidade excessiva de registros de artigos. Após algumas rodadas chegou-se a string utilizada neste trabalho que está apresentada na tabela 2 .

Tabela 2. Componentes do protocolo da busca

\begin{tabular}{|c|c|c|c|}
\hline Método de busca selecionado & Bibliotecas & Idioma selecionado & String de busca \\
\hline $\begin{array}{l}\text { Busca em publicações indexadas por } \\
\text { bibliotecas digitais conveniadas com } \\
\text { o Portal da CAPES }\end{array}$ & $\begin{array}{l}\text { SCOPUS; } \\
\text { WEB OF } \\
\text { SCIENCE; }\end{array}$ & $\begin{array}{l}\text { Inglês, idioma padrão } \\
\text { utilizado nas } \\
\text { bibliotecas digitais }\end{array}$ & $\begin{array}{l}\text { Keyword: ("leadership" OR } \\
\text { "success factors") AND "software } \\
\text { process improvement" }\end{array}$ \\
\hline
\end{tabular}

\footnotetext{
${ }^{2}$ www.scopus.com

${ }^{3}$ http://ip-science.thomsonreuters.com/pt/produtos/wos/
} 
Para a seleção dos artigos, foram adotados os critérios de inclusão e exclusão apresentados na Tabela 3.

Tabela 3.Critérios de inclusão e exclusão de artigos na pesquisa

\begin{tabular}{|l|l|}
\hline Tipo de critério & Critério \\
\hline Inclusão & Artigos que abordem a liderança em iniciativas de melhoria de processo de software \\
\hline \multirow{2}{*}{ Exclusão } & Artigos inacessíveis ou indisponíveis para pesquisa. \\
\cline { 2 - 2 } & Artigos que não abordem a liderança em iniciativas de melhoria de processo de software. \\
\hline
\end{tabular}

Durante a fase de execução, a partir das palavras-chave apresentadas na tabela 2, foram encontrados inicialmente 17 artigos na biblioteca digital Web of Science e 173 artigos na biblioteca SCOPUS. Assim, nesta fase o total de artigos retornados foi de 190. Num primeiro momento, o processo de seleção considerou títulos e resumos para realizar a filtragem de acordo com os critérios estabelecidos na tabela 3. Após o primeiro filtro, restaram 14 artigos sendo que apenas dois artigos não estavam acessíveis, restando 12 artigos para o estudo. Estes passaram no segundo filtro que consistia na leitura integral do artigo. Nesta fase da revisão, excluíram-se os artigos que não faziam menção à liderança em iniciativas de melhoria de processo de software. Após essa fase, restaram três artigos: (1) An empirical investigation of the key factors for success in software process improvement [Dyba, 2005], (2) Key success factors for implementing software process improvement: a maturity-based analysis [Rainer e Hall, 2002] e (3) Quantitative assessments of key success factors in software process improvement for small and medium web companies [Sulayman e Mendes, 2010]. A Tabela 4 apresenta os trabalhos selecionados a partir das bibliotecas digitais para a pesquisa, ordenados pela data de publicação. A pesquisa foi realizada no início do segundo semestre de 2011.

Tabela 4. Relação dos dados dos artigos encontrados nas bases pesquisadas

\begin{tabular}{|c|c|c|c|c|c|}
\hline Autores & Títulos dos Trabalhos & Fonte da Publicação & Ano & $\begin{array}{l}\text { Cita- } \\
\text { ções }\end{array}$ & $\begin{array}{l}\text { Biblioteca } \\
\text { digital }\end{array}$ \\
\hline $\begin{array}{l}\text { Rainer A, } \\
\text { Hall T }\end{array}$ & $\begin{array}{l}\text { Key success factors for implementing } \\
\text { software process improvement: a maturity- } \\
\text { based analysis }\end{array}$ & $\begin{array}{l}\text { Journal of Systems } \\
\text { and Software }\end{array}$ & 2002 & 49 & $\begin{array}{l}\text { SCOPUS } \\
\text { e Web of } \\
\text { Science }\end{array}$ \\
\hline Dybå, T. & $\begin{array}{l}\text { An empirical investigation of the key factors } \\
\text { for success in software process } \\
\text { improvement }\end{array}$ & $\begin{array}{l}\text { IEEE Transactions } \\
\text { on Sw.Engineering }\end{array}$ & 2005 & 53 & $\begin{array}{l}\text { SCOPUS } \\
\text { e Web of } \\
\text { Science }\end{array}$ \\
\hline $\begin{array}{l}\text { Sulayman, } \\
\text { M } \\
\text { Mendes, E. }\end{array}$ & $\begin{array}{l}\text { Quantitative assessments of key success } \\
\text { factors in software process improvement for } \\
\text { small and medium web companies }\end{array}$ & $\begin{array}{l}\text { SAC '10 ACM } \\
\text { Symposium on } \\
\text { Applied Computing }\end{array}$ & 2010 & 0 & $\begin{array}{l}\text { SCOPUS } \\
\text { e Web of } \\
\text { Science }\end{array}$ \\
\hline
\end{tabular}

Esta pesquisa ainda considerou artigos encontrados com base em pesquisa manual nos anais do Simpósio Brasileiro de Qualidade de Software, edição 2011. Por não estar disponível uma biblioteca digital contendo as publicações do SBQS permitindo uma pesquisa automatizada a busca realizada neste trabalho foi planejada apenas nas publicações de 2011. Assim, foram selecionados mais dois artigos para o estudo: (1) Uma Investigação sobre os Fatores Críticos de Sucesso em Iniciativas de Melhoria de Processos de Software [Montoni e Rocha, 2011] e (2) Programas de Melhoria de Processo de Software - Uma pesquisa sobre a influência dos aspectos humanos [Santos et. al., 2011]. Como procedimentos para extração das informações 
relevantes para a pesquisa, cada um dos cinco artigos selecionados para o estudo tiveram suas referências bibliográficas armazenadas e foram examinados a fim de identificar a visão dos autores sobre a influência da liderança nas iniciativas de melhoria de processo de desenvolvimento de software. Neste sentido, durante a análise dos artigos estudados, o entendimento por influência da liderança nas iniciativas de MPS foi realizado com base na comparação dos fatores de sucesso discutidos pelos autores dos artigos analisados com as características dos papéis do líder organizacional definidos por Quinn (2003). Estas informações foram coletadas, sumarizadas, armazenadas e estão disponibilizadas nas próximas seções deste artigo.

\section{Apresentação e Análise dos Artigos Selecionados}

Recentemente, a investigação para a compreensão da liderança vem apresentando interesse, tanto por parte das organizações quanto da academia. Esse interesse pode ser evidenciado observando a expressiva quantidade de artigos científicos que são publicados discutindo conceitos de liderança, assim como resultados de investigações científicas conduzidas recentemente e diretamente relacionadas com o tema [Gourlay, 2006; 1996 apud Krogh; Nonaka e Rechsteiner, 2011] [Nonaka e Takeuschi, 2011]. Com o intuito de contribuir com a pesquisa para a compreensão da liderança voltando às atenções especificamente para a área de MPS este trabalho apresenta o resultado da análise de cinco artigos selecionados pelo mapeamento sistemático. Os trabalhos estudados são discutidos a seguir considerando a ordem cronológica de publicação.

\subsection{Artigo: Key success factors for implementing software process improvement: a maturity-based analysis [Rainer e Hall, 2002]}

Neste trabalho os autores realizam uma investigação baseada em quatro perguntas de pesquisa:

- Quais os fatores que os entrevistados pensam ter grande impacto na implementação de MPS?

- Quais os fatores que os entrevistados pensam não ter impacto na implementação de MPS?

- Não é possível diferenciar os fatores que são percebidos por entrevistados de organizações de alta e baixa maturidade?

- Existem diferenças nos fatores reconhecidos pelos entrevistados entre empresas com sucesso e sem sucesso em iniciativas de MPS?

Os fatores de sucesso pesquisados neste trabalho foram identificados durante a fundamentação teórica da pesquisa. Assim, os fatores são os mesmos usados em outras pesquisas como as consideradas nos estudos [El Emam, et. Al., 2001; Goldenson e Herbsleb, 1995; Stelzer e Mellis, 1998 apud Rainer e Hall, 2002].

A investigação considerou empresas com diferentes níveis de maturidade: (a) empresas avaliadas formalmente por equipes externas, (b) empresas avaliadas internamente (auto-avaliação) e (c) aquelas ainda sem a maturidade avaliada. Quatro fatores de sucesso foram reconhecidos por todas as empresas como fatores de grande impacto sobre a MPS: (1) revisões em processos; (2) normas e procedimentos; (3) treinamento e mentoring; (4) pessoal experiente. As empresas com baixa maturidade 
envolvidas na pesquisa consideraram que apenas dois fatores tiveram um grande impacto na MPS: (1) normas e procedimentos e (2) treinamento e mentoring. Já as empresas mais maduras envolvidas na pesquisa consideraram ter um grande impacto na MPS quatro fatores principais: (1) liderança interna; (2) inspeções; (3) apoio executivo e (4) apropriação de processos internos. Os autores do artigo estudado identificaram dois fatores que não tem impacto sobre a MPS: (1) ferramentas de avaliação e (2) esquemas de recompensa. A partir do resultado obtido com o estudo realizado, quatro fatores mais amplos emergiram como recomendações para profissionais e pesquisadores de melhoria de processo de software: (1) processo; (2) pessoas; (3) competências e (4) liderança.

Estes fatores sugerem que melhoria de processos pode ser mais eficaz se ocorrer em conjunto com o desenvolvimento de competências do pessoal envolvido. Portanto, neste trabalho, os autores concluem que a liderança tem destaque como fator crítico de sucesso para a melhoria de processos de software, mas não se aprofundam mais sobre aspectos relacionados à liderança.

\subsection{Artigo: An empirical investigation of the key factors for success in software process improvement [Dyba, 2005]}

Dos cinco trabalhos analisados, esta é a obra mais citada com 53 citações na SCOPUS. Neste trabalho, o autor apresenta uma pesquisa empírica dos fatores-chave para o sucesso nas iniciativas de MPS. Para Dybå (2005), a compreensão de como implementar a MPS com sucesso é uma questão ainda em aberto. Ele considera que a literatura contém, na sua grande maioria, estudos de caso de empresas que descrevem suas iniciativas e programas de melhoria, sendo que estas são cientificamente limitadas e inconclusivas. Assim, ele justifica seu trabalho fazendo um levantamento quantitativo de 120 organizações de software para testar o modelo conceitual e hipóteses do seu estudo. Os resultados deste trabalho indicam que o sucesso da MPS depende criticamente de seis fatores organizacionais que foram utilizados como variáveis independentes da pesquisa conduzida por Tore Dybå (2005):

- Orientação de negócios: medida sobre quanto metas e ações de MPS estão alinhadas com metas e estratégias de negócio explícitas e implícitas.

- Envolvimento dos líderes: medida sobre o real grau de comprometimento e participação dos líderes em MPS, considerando todos os níveis da organização.

- Participação dos funcionários: medida sobre até que ponto funcionários usam seu conhecimento e experiência para decidir, agir e assumir a responsabilidade pela MPS.

- Preocupação com a medição: medida sobre quanto a organização coleta e utiliza dados de qualidade para orientar e avaliar os efeitos de atividades MPS.

- Exploração do conhecimento existente: medida sobre o grau de envolvimento da organização na exploração do conhecimento existente.

- Exploração de novos conhecimentos: medida sobre o grau de envolvimento da organização na exploração de novos conhecimentos. 
Neste trabalho, as seis variáveis independentes supracitadas conseguem explicar mais de $50 \%$ da variação do sucesso nas iniciativas de MPS. Dybå (2005) também desenhou um conjunto de sete hipóteses para seu estudo: (1) o sucesso nas iniciativas de MPS está positivamente associado com a orientação de negócios; (2) o sucesso nas iniciativas de MPS está positivamente associado ao envolvimento dos líderes; (3) o sucesso nas iniciativas de MPS está positivamente associado com a participação dos funcionários; (4) o sucesso nas iniciativas de MPS está positivamente associado com preocupação com a medição; (5) o sucesso nas iniciativas de MPS está positivamente associado com a exploração do conhecimento existente; (6) o sucesso nas iniciativas de MPS está positivamente associado com a exploração de novos conhecimentos e (7) As seis variáveis explicam uma grande quantidade de variância do sucesso nas iniciativas de MPS.

A variável independente (Envolvimento dos líderes) e a hipótese (o sucesso nas iniciativas de MPS está positivamente associada com o envolvimento dos líderes) foram consideradas na pesquisa de Dybå (2005) por que um tema predominante na gestão da qualidade é a importância do comprometimento da liderança [Ahire, Golhar e Waller, 1996; Black e Porter, 1996; Powell, 1995; Saraph, Benson e Schroeder, 1989; Yusof e Aspinwall, 1999 apud Dybå , 2005] e seu envolvimento na implementação de ações de melhoria [El Emam, et. Al., 2001; Goldenson e Herbsleb, 1995; Humphrey, 1989; Stelzer e Mellis, 1998; Zahran, 1998 apud Dybå , 2005].

O envolvimento dos líderes é de suma importância para a MPS. Para a construção de organizações de software com capacidades de aprendizagem, é necessário haver liderança em todos os níveis envolvidos na MPS que estejam empenhados em aprender e melhorar os processos, e que transmitam a responsabilidade para os demais envolvidos na iniciativa de MPS [Dybå , 2005]. A publicação de Dybå (2005) mostra as médias, desvios-padrão, e 15 correlações entre as variáveis independentes, onde duas apresentam um coeficiente de correlação maior que 0,5 . A correlação com o coeficiente mais alto $(0,63)$ está entre as variáveis (1) Orientação de negócios e (2) Envolvimento da liderança. Assim, "O envolvimento da liderança", medida sobre o real grau de comprometimento e participação dos líderes em MPS, considerando todos os níveis da organização, apresentou uma correlação significativa com o sucesso das iniciativas de MPS. Isto suporta a hipótese de que o sucesso nas iniciativas de MPS é positivamente associado à liderança envolvida. Neste contexto, Dybå (2005) considera que sem indivíduos que aprendem, não pode haver melhoria. Deste modo, o papel fundamental dos gerentes e líderes durante as iniciativas de melhoria de processos é estabelecer e manter contextos organizacionais que favoráveis à aprendizagem organizacional e MPS.

Dybå (2005) sugere que, ao invés de focar as atividades de MPS em procedimentos técnicos, as organizações de software devem concentrar seus esforços de MPS também em criar uma cultura organizacional onde a MPS possa prosperar. Isto difere substancialmente do que vem sendo publicado nas principais literaturas de MPS já publicadas, pois estas se concentram quase exclusivamente em ferramentas de Engenharia de Software e técnicas. Uma visão nova e importante apresentada na pesquisa de Dybå (2005) é que as questões organizacionais são pelo menos tão importante quanto a tecnologia para as iniciativas de MPS. Portanto, a pesquisa foca nos aspectos quantitativos e não aprofunda a pesquisa com intuito de discutir questões relacionadas à influência da liderança nas iniciativas de MPS. 


\subsection{Artigo: Quantitative assessments of key success factors in software process improvement for small and medium web companies [Sulayman e Mendes, 2010]}

Neste trabalho, os autores apresentam o resultado de uma avaliação quantitativa dos fatores de sucesso para pequenas e médias empresas que desenvolvem software exclusivamente para a web. Foram coletados dados de 20 empresas e a pesquisa contou com 72 respostas individuais. Os autores aplicaram o mesmo modelo teórico dos fatores de sucesso de iniciativas de MPS, técnicas e questionário de coleta de dados propostos e empregados em (Dybå, 2005). A principal diferença com o estudo de Dybå (2005), foi que a amostra de organizações considerou apenas pequenas e médias empresas que desenvolvem exclusivamente para a web. Foram testadas todas as seis variáveis e as sete hipóteses associadas do modelo teórico proposto por Dybå (2005).

Como resultado, constatou-se que há vários pontos de convergência entre ambos os estudos. No entanto, houve também diferenças este eles. Contrariamente às conclusões Dybå (2005), as variáveis A Liderança Envolvida e Exploração do Conhecimento Existente mostraram maior correlação com A Orientação de Negócios. Como em Dybå (2005) foi realizada uma análise de regressão para descobrir qual subconjunto de fatores que influenciaram o sucesso em iniciativas de MPS de forma mais significativa. Neste trabalho A Liderança Envolvida foi o fator mais influente, contribuindo para o sucesso de $26 \%$ nas iniciativas de MPS quando considerada separadamente das outras variáveis independentes. A Participação dos Funcionários, juntamente com A Liderança Envolvida contribuíram com 30\%. A Preocupação com Medição também mostrou um valor significativo em ambos os modelos de regressão e, portanto, também contribuiu para o sucesso da MPS em geral.

Os resultados mostraram que todas as variáveis independentes contribuíram para o sucesso na MPS. Entretanto, A orientação de negócios, A Exploração do conhecimento existente e A Exploração de novos conhecimentos contribuíram parcialmente para o sucesso da MPS. Já A Liderança envolvida, A participação dos funcionários e A Preocupação com a medição contribuíram e deram um forte apoio para o sucesso da iniciativa em MPS. Neste trabalho os autores enfatizam o estudo em identificar, mas não em compreender e tentar explicar como se dá o envolvimento da liderança nas iniciativas de MPS estudadas.

\subsection{Artigo: Programas de Melhoria de Processo de Software - Uma pesquisa sobre a influência dos aspectos humanos [Santos et. al., 2011]}

Este artigo apresenta uma pesquisa conduzida em três empresas de software amazonenses e teve como propósito aprofundar a compreensão sobre aspectos humanos que influenciam um programa de MPS. Nesta pesquisa foram verificadas 14 dos aspectos humanos advindos do comportamento organizacional descrito em Robbins (2005) apud [Santos et al., 2011]. Entre estes, foi verificado o aspecto da Eficácia da Liderança. Segundo Santos (2011) os colaboradores das organizações envolvidas na pesquisa consideraram que houve liderança efetiva no programa de MPS. Isto se deve ao fato de pessoas capacitadas estarem envolvidas para executar com exclusividade as atividades necessárias. Quanto à Eficácia de Liderança os autores sugerem que é necessário "verificar a capacidade de liderança do responsável pelo programa de MPS na organização sob pena de não se obter êxito na implementação do programa de melhoria de processos" [Santos et. al, 2011]. Cabe ressaltar que nesta pesquisa também 
foi observado que a "dedicação exclusiva do responsável pelo MPS foi importante para a execução das atividades" [Santos et. al, 2011].

Baseado nos resultados da pesquisa, os autores apresentam um conjunto de sugestões que profissionais poderiam considerar em futuros programas de melhoria de processo de software: (1) processo de aprendizagem, (2) treinamentos, (3) motivação, (4) satisfação com o trabalho, (5) personalidade, (6) eficácia da liderança, (7) tomada de decisões, (8) avaliação de desempenho, (9) estresse profissional e (10) percepção do programa de MPS. Pode-se observar que grande parte das sugestões apresentadas estão diretamente relacionadas ao papel do líder nas organizações como definido por Quinn (2003). Contudo o trabalho não enfatiza o estudo para compreender a influência do líder organizacional nos programas de MPS.

\subsection{Artigo: Uma Investigação sobre os Fatores Críticos de Sucesso em Iniciativas de Melhoria de Processos de Software [Montoni e Rocha, 2011]}

Neste trabalho os pesquisadores realizaram uma investigação sobre fatores críticos de sucesso em iniciativas de MPS. As seguintes questões de pesquisa foram definidas para este estudo: (1) Quais são os fatores capazes de influenciar o sucesso de iniciativas de melhoria? e (2) Como os implementadores de melhoria tratam os fatores capazes de influenciar o sucesso de iniciativas de melhoria? A pesquisa foi realizada em duas etapas. Inicialmente foi conduzido um survey com 25 implementadores de MPS e membros de diferentes organizações de software, além de uma revisão sistemática da literatura para investigar fatores críticos de sucesso da MPS. Com o objetivo de determinar o grau de influência dos fatores críticos de sucesso identificados, a segunda etapa da pesquisa foi realizada aplicando outro survey e entrevistas semi-estruturadas com implementadores de MPS com vasta experiência e que tivessem participado em iniciativas de MPS bem sucedidas. Como resultado o conjunto de categorias de fatores críticos de sucesso foi estendida, inter-dependências entre as categorias foram definidas, além um conjunto de hipóteses ter sido elaborado com o objetivo de descrever relacionamentos entre as categorias. Estes resultados permitiram a construção de um framework teórico, que tem como propósito explicar o processo social que norteia as iniciativas de MPS.

Dos 25 fatores críticos de sucesso estudado pelos autores deste artigo, 5 se destacaram como fatores de maior criticidade para o sucesso de iniciativas de melhoria, por aparecerem em mais de $50 \%$ das ocorrências, tanto no survey quanto na revisão sistemática [Montoni e Rocha, 2011]: (1) Competências em engenharia de software dos membros da organização (conhecimento, experiências e habilidades); (2) Apoio efetivo da alta gerência; (3) Existir gerência do projeto de implementação da melhoria dos processos (4) Adequação dos processos/procedimentos definidos e (5) Conscientização dos membros da organização quanto aos benefícios obtidos com a implantação dos processos. Analisando estas 5 propriedades, observa-se que todas são diretamente relacionadas com a capacidade de influenciar pessoas para atingir um propósito comum, conforme o conceito de liderança descrito por [Northouse, 2004] e ao papel do líder nas organizações como definido por Quinn (2003). Embora este artigo apresente um trabalho científico que teve como principal objetivo desenvolver o framework teórico para explicar o processo social que existe nas iniciativas de MPS os autores não focaram 
no aprofundamento dos conhecimentos especificamente sobre a influência do líder organizacional nos programas de MPS.

\subsection{Análise dos Artigos estudados}

Com o resultado do mapeamento sistemático realizado nesta pesquisa foi elaborada a tabela 5 para apoiar a análise dos artigos selecionados para o trabalho. A tabela apresenta um relacionamento dos fatores críticos de sucesso mais relevantes relatados por cada um dos artigos estudados com as seis categorias dos papeis do líder organizacional descritos por Quinn (2003). A primeira versão deste relacionamento foi construída pela pesquisadora e validada por meio de um workshop realizado durante o Seminário de Liderança que é conduzido semanalmente pelo grupo de pesquisa do Laboratório de Liderança \& Gestão Responsável (LGR) do Programa de Pós-Graduação em Engenharia e Gestão do Conhecimento (EGC). O relacionamento envolveu tanto especialistas em Liderança quanto em MPS que em determinados momentos foram convocados a colaborar com suas experiências sobre o problema tratado. O workshop também contou com o envolvimento de um facilitador que motivou o diálogo com os participantes durante sua realização.

Tabela 5 Análise comparativa dos artigos selecionados para a pesquisa

\begin{tabular}{|c|c|}
\hline Autores & Fatores de sucesso enfatizados vs. Papel do Líder (Quinn, 2003) \\
\hline $\begin{array}{l}\text { Rainer A, } \\
\text { Hall T }\end{array}$ & $\begin{array}{l}\text { 1.Processo: Realizador de Metas/ Monitorador / Inovador } \\
\text { 2.Pessoas: Mentor } \\
\text { 3.Competências: Mentor } \\
\text { 4.Liderança: Realizador de Metas / Mentor / Facilitador / Monitorador / Intermediador / Inovador }\end{array}$ \\
\hline Dybå, T. & $\begin{array}{l}\text { 1.Orientação de negócios: Intermediador / Facilitador } \\
\text { 2.Envolvimento dos líderes: Realizador de Metas / Mentor / Facilitador / Monitorador / } \\
\text { Intermediador / Inovador } \\
\text { 3.Participação dos funcionários: Mentor / Facilitador } \\
\text { 4.Preocupação com a medição: Monitorador } \\
\text { 5.Exploração do conhecimento existente: Mentor } \\
\text { 6.Exploração de novos conhecimentos: Mentor / Inovador / Realizador de Metas }\end{array}$ \\
\hline $\begin{array}{l}\text { Sulayman, M } \\
\text { Mendes, E. }\end{array}$ & $\begin{array}{l}\text { 1.Orientação de negócios: Intermediador / Facilitador } \\
\text { 2.Envolvimento dos líderes: Realizador de Metas / Mentor / Facilitador / Monitorador / } \\
\text { Intermediador / Inovador } \\
\text { 3.Participação dos funcionários: Mentor / Facilitador } \\
\text { 4.Preocupação com a medição: Monitorador } \\
\text { 5.Exploração do conhecimento existente: Mentor } \\
\text { 6. Exploração de novos conhecimentos: Mentor / Inovador / Realizador de Metas }\end{array}$ \\
\hline $\begin{array}{l}\text { Santos et. Al., } \\
2011\end{array}$ & $\begin{array}{l}\text { 1.Processo de aprendizagem: Mentor / Realizador de Metas / Facilitador } \\
\text { 2. Treinamentos: Mentor } \\
\text { 3. Motivação: Realizador de Metas / Mentor / Facilitador / Monitorador / Intermediador } \\
\text { 4.Satisfação com o trabalho: Mentor } \\
\text { 5.Personalidade: Mentor / Intermediador } \\
\text { 6.Eficácia da liderança: Realizador de Metas / Mentor / Facilitador / Monitorador / } \\
\text { Intermediador } \\
\text { 7.Tomada de decisões: Realizador de Metas / Mentor / Facilitador / Monitorador } \\
\text { 8.Avaliação de desempenho: Monitorador } \\
\text { 9.Estresse profissional: Mentor } \\
\text { 10.Percepção do programa de MPS: Intermediador / Mentor }\end{array}$ \\
\hline $\begin{array}{l}\text { Montoni e } \\
\text { Rocha } 2011\end{array}$ & $\begin{array}{l}\text { 1.Competências em engenharia de software: Mentor } \\
\text { 2.Apoio efetivo da alta gerência: Realizador de Metas / Mentor / Facilitador } \\
\text { 3. Existir gerência do projeto de implementação: Monitorador } \\
\text { 4. Adequação dos processos/procedimentos: Realizador de Metas/ Monitorador / Inovador } \\
\text { 5.Conscientização dos membros da organização quanto aos benefícios obtidos com a MPS: } \\
\text { Intermediador / Mentor }\end{array}$ \\
\hline
\end{tabular}


Analisando os dados da tabela 5 pode-se perceber que embora os artigos selecionados para a pesquisa tenham investigado diferentes fatores de sucesso em programas de MPS, todos os artigos elencam em média um conjunto de 5 a 7 fatores de sucesso mais relevantes para as iniciativas de MPS.

Respondendo a questão secundária de pesquisa 1: Quais categorias dos papeis do líder organizacional descritos por Quinn (2003) tem relação com fatores de sucesso em iniciativas de melhoria de processo de software? Os resultados da pesquisa permitem observar que todos os fatores de sucesso mais relevantes para as iniciativas de melhoria de processo de desenvolvimento de software, que foram identificados neste estudo, se relacionam com ao menos uma das seis categorias dos papeis do líder organizacional descritos por Quinn (2003).

Em resposta à questão secundária de pesquisa 2: Qual é a visão dos pesquisadores que investigam sobre fatores de sucesso em iniciativas de melhoria de processo de software quando avaliam a liderança em tais iniciativas? Como resultado pode-se constatar que os autores de todos os trabalhos estudados discutem aspectos relacionados com o papel do líder organizacional como um dos principais, senão o principal, fator de sucesso para as iniciativas de MPS sobressaindo-se aos demais.

Respondendo a questão de pesquisa principal: Qual a influência da liderança nas iniciativas de melhoria de processo de desenvolvimento de software? Considerando que o entendimento por influência da liderança nas iniciativas de MPS foi realizado com base na comparação dos fatores de sucesso discutidos pelos autores dos artigos analisados com as categorias dos papéis do líder organizacional definidos por Quinn (2003), o resultado desta pesquisa demonstra que existe uma tendência de que os fatores humanos e sociais que influenciam nas iniciativas de MPS estão predominantemente relacionados a aspectos da liderança organizacional.

Contudo os artigos selecionados para a pesquisa não se aprofundam especificamente na compreensão da influência da liderança organizacional nas iniciativas de MPS. Com esta pesquisa foi possível verificar que existe espaço para uma investigação especificamente sobre a influência da liderança em iniciativas de MPS que poderia contribuir também para a expansão da literatura sobre a liderança considerando os elementos envolvidos na teoria da criação do conhecimento organizacional [Nonaka e Takeushi, 1997].

\section{Considerações Finais}

Este artigo apresentou o resultado de um mapeamento sistemático de artigos científicos com foco na liderança em iniciativas de MPS. Tais iniciativas envolvem mudanças organizacionais e os reflexos da liderança nestes programas devem ser entendidos para apoiar a melhoria de sua gestão e do grau de eficácia da iniciativa como um todo. Entretanto, durante uma pesquisa bibliográfica exploratória, não foram encontrados estudos sistemáticos sobre a liderança em iniciativas de melhoria. Desta forma, a contribuição deste trabalho foi a realização de um mapeamento de estudos, utilizando técnicas de revisão sistemática da literatura, para identificar publicações relevantes sobre programas de melhoria de processo de software que trouxessem discussões abordando a liderança envolvida em tais iniciativas. 
Como resultado, apenas 3 artigos foram selecionados de 14 recuperados. A pesquisa ainda considerou trabalhos relacionados com o tema que também tivessem sido conduzidos e publicados no SBQS 2011. Assim, mais 2 artigos foram selecionados.

Todos os trabalhos analisados consideram a liderança como um fator de sucesso nas iniciativas de melhoria de processo de software. Este trabalho também identificou que todos os fatores de sucesso mais relevantes encontrados nos artigos selecionados se relacionam diretamente com pelo menos uma das seis categorias do papel do líder organizacional proposto por Quinn (2003).

Como resultado desta pesquisa pode-se constatar a relevância do comprometimento da liderança envolvida, sobressaindo-se aos demais fatores considerados para o êxito dos programas de melhoria de processos de software. Uma visão ainda atual e importante apresentada pelos trabalhos estudados é que, mesmo as questões organizacionais, humanas e sociais sendo tão importantes quanto os aspectos técnicos para as os programas de melhoria de processo, estas questões ainda são pouco exploradas pelas pesquisas em MPS.

Esta pesquisa também apresenta como resultado a evidenciação da ausência de trabalhos científicos publicando resultado de pesquisas que abordem exclusivamente a questão da liderança em programas de melhoria de processos de software. Sobretudo discutindo aspectos relacionados à liderança em programas de MPS associados com os componentes da Teoria da Criação do Conhecimento Organizacional. Deste modo, pode-se observar que existe uma lacuna que poderia ser preenchida com estudos específicos nesta área visando contribuir para a compreensão e melhoria da gestão de iniciativas de MPS.

Os autores deste artigo pretendem expandir o resultado da pesquisa buscando publicações relacionadas ao tema investigado em diferentes edições do SBQS e em outras conferências relevantes para esta pesquisa. Ainda como trabalhos futuros os autores deste trabalho irão desenvolver um projeto de pesquisa com empresas que foram avaliadas com sucesso nas iniciativas de MPS buscando compreender e explicitar a influência da liderança nas iniciativas de MPS. Como resultado se espera estabelecer um referencial teórico que suporte os papéis do líder organizacional nas diferentes etapas de programas MPS nos casos que serão estudados. Assim se espera contribuir tanto para a ampliação do conhecimento científico sobre a liderança no processo de criação do conhecimento organizacional quanto para o progresso das iniciativas MPS.

\section{Agradecimentos}

Os autores gostariam de agradecer aos pesquisadores do Laboratório de Liderança \& Gestão Responsável (LGR) do Programa de Pós-Graduação em Engenharia e Gestão do Conhecimento (EGC) que participaram do workshop de validação do relacionamento dos fatores de sucesso mais relevantes relatados por cada um dos artigos estudados com as seis categorias dos papeis do líder organizacional descritos por Quinn (2003). 


\section{Referências}

Alvesson, M., Knowledge work and knowledge-intensive firms, Joumal of Management Studies, 271 páginas, Oxford UK, 2004

Card, D. N. (2004), "Research Directions in Software Process Improvement", In: 28th COMPSAC'2004, Hong Kong, China, IEEE Computer Society, p. 238, 27-30.

Dybå, T. An Empirical Investigation of the Key Factors for Success in SPI. 2005. IEEE Trans. of Software Eng. 31, 5, 410-424.

Fialho, F., Macedo, M., Santos, N. dos, Mitidieri, T. C., Gestão do conhecimento organizacional, Editora UFSC, 2010

Kitchenham, B., Procedures for Performing Systematic Reviews, Technical Report, Departament of Computer Science Keele University, Keele, 2004

Kotter, J. Liderando Mudança. Rio de Janeiro: Campus, 1997, 188p.

Krogh, G. von; Nonaka, I.; e Rechsteiner, L.. Leadership in Organizational Knowledge Creation: A Review and Framework. Journal of Management Studies, p.1-38, 2011.

Montoni, M. A., Lições Aprendidas com Implementação do Modelo MPS em Empresas MPS.BR, Lições Aprendidas SOFTEX, p. 32-44, 2008 Acesso em 05 de julho de 2011 e disponível em http://www.softex.br/mpsbr/_livros/licoes/mpsbr_pt.pdf

Montoni, m. a., Rocha, a. r. c., Uma Investigação sobre os Fatores Críticos de Sucesso em Iniciativas de Melhoria de Processos de Software In: X Simpósio Brasileiro de Qualidade de Software (SBQS 2011), Curitiba, PR, p. 151-165, 2011.

Niazi, M., Wilson, D., Zowghi, D., 2006, Critical success factors for software process improvement implementation: An empirical study, Software Process Improvement and Practice, v. 11, n. 2, pp. 193-211.

Nonaka, I. \& Takeuchi, H.. Criação do conhecimento na empresa. Rio de Janeiro: Campus, 1997.

Nonaka, I.; Takeuchi, H.. The wise leader. Harvard business review, vol. 89, no. 5, p. 58-67, 146, 2011.

Northouse, P. G., Leadership: Theory and practices. Sage Publications, Inc.:3 ed. 2004

Quinn, R.E. et al. Competências Gerenciais: princípios e aplicações. Rio de Janeiro: Elsevier, 2003.

Rainer, A., Hall, T., 2003, A quantitative and qualitative analysis of factors affecting software processes, Journal of Systems and Software, v. 66, n. 1, pp. 7-21.

Rainer, A., Hall, T., 2002, Key Success Factors for Implementing Software Process Improvement: A Maturity-Based Analysis, J. Systems and Software, vol. 62, no. 2, pp. 71-84.

Santos, D. V., Vilela, D., Souza, c., Conte, T. Programas de Melhoria de Processo de Software - Uma pesquisa sobre a influência dos aspectos humanos In: X Simpósio Brasileiro de Qualidade de Software (SBQS 2011), Curitiba, PR, p. 183-197, 2011 
Senge, P. "A quinta disciplina", acessado em 26 de agosto de 2011; disponível em: http://www.softwarepublico.gov.br/file/16685703/quintasenge.pdf

SEI, CMMI-DEV SCAMPI Class A Appraisal Results 2010 Mid-Year Update Sep 2010, acessado em 26 de agosto de 2011; disponível em: http://www.sei.cmu.edu/cmmi/casestudies/profiles/pdfs/upload/2010SepCMMI.pdf

SEI, CMMI SCAMPI Class A Appraisal Results 2011 End-Year Update Mar 2012, acessado em 30 de abril de 2012; disponível em: http://www.sei.cmu.edu/cmmi/casestudies/profiles/pdfs/upload/2012MarCMMI.pdf

SOFTEX, Relatório de Avaliações MPS Publicadas, acessado em 30 de abril de 2012; disponível em: http://www.softex.br/mpsbr/_avaliacoes/avaliacoes_mpsbr_total.pdf

Sulayman, M., Mendes, E. Quantitative assessments of key success factors in software process improvement for small and medium web companies, The University of Auckland in: SAC '10 Proceedings of the 2010 ACM Symposium on Applied Computing.

Swart, J., Kinnie, N., Sharing knowledge in knowledge-intensive firms, Human Resource Management Journal, Volume 13, Issue 2, pages 60-75, April ACM New York, NY, USA C2010

Travassos, G., Kalinowski, M., 2010, "Resultados iniciais do iMPS 2010: Variação de Desempenho nas Empresas que Adotaram o Modelo MPS". In: VI Workshop Anual do MPS, pp. 24-33, Campinas, SP - Brasil. 